\title{
The Effects of the Solvent Choice of the Continuous Phase on the Poly(Urea-Urethane) Microcapsules Properties
}

\author{
F. Salaün*
}

\author{
Univ. Lille, ENSAIT, GEMTEX-Laboratoire de Génie et Matériaux Textiles, F-59000 Lille, France
}

\begin{abstract}
Xylitol, a natural crystalline polyol, presents a cooling effect due to its negative heat of solution at $35{ }^{\circ} \mathrm{C}$ supported by humidity absorption, contributing to a fresh sensation when it dissolves. Since this material is sometimes in a liquid state, it cannot be incorporated in or onto a substrate without being protected. One of the strategies to protect the active substance may be forming a barrier layer at its surface, i.e., microencapsulation. The present work is devoted to studying the effect of continuous phase parameters affecting on encapsulation of xylitol with a poly (urea-urethane) shell through a two-step microencapsulation process. The first step is liquid-liquid dispersion either in toluene or Miglyol $812 \mathrm{~N}$, and the second step is microencapsulation by interfacial polymerization. The process can be used to control the size distribution of the microparticles, the thickness, and the chemical nature of the shell, which influences the release rate of the active substance. The choice of the continuous phase solvent (toluene or Miglyol $812 \mathrm{~N}$ ) required some changes in the formulation of the system, especially the HLB of the surfactant mixture, to obtain a stable emulsion with a narrow particle size distribution. The thermo-chemical and morphological characteristics of microparticles were studied by Fourier transform-infrared spectroscopy (FT-IR), differential scanning calorimetry (DSC), enthalpy of dilution, and scanning electron microscope (SEM). The microparticle size is governed by the emulsion step and the chemical composition of the organic phase. Most of the thermal properties are related to their porous structure and their chemical shell formation during the interfacial polymerization step.
\end{abstract}

Keywords: Microencapsulation, Polyurethane shell, Water in oil emulsion, Porous shell, Thermal properties.

\section{INTRODUCTION}

Microencapsulation has been recognized as a helpful tool to protect sensitive active substances allowing to implement it on or in various substrates to design smart materials [1]. This technology allows the synthesis of tiny particles, which find their applications in various industrial fields [2]. Nevertheless, for a textile application, the particles need to meet several criteria allowing their implementation, i.e., a narrow particle size distribution, a mean diameter between 1 to $15 \mu \mathrm{m}$ to avoid any breakage during the finishing treatment suitable thermo-mechanical properties [3]. In recent years, research efforts have been made to design cooling textile structures that interact with the microclimate conditions between the skin of the wearer and the first textile layer [4]. This textile type improves comfort when worn and interacts with the excess sweat of the user in hot conditions or physical effort [5]. The principle is based on the diffusion of the humidity within the microparticles, the latter act as a microreactor [6]. These particles store the sweat in the liquid form to react chemically with the active ingredient [7].

As a natural polyol, xylitol has a cooling effect due to its negative heat of solution at $35 \circ \mathrm{C}\left(-36.5 \mathrm{kcal} \cdot \mathrm{kg}^{-1}\right)$ supported by humidity absorption, contributing to a

"Address correspondence to this author at the Univ. Lille, ENSAIT, GEMTEX-Laboratoire de Génie et Matériaux Textiles, F-59000 Lille, France; E-mail: fabien.salaun@ensait.fr fresh sensation when it reacts with water [8]. The encapsulation step allows protecting this active compound, whatever its physical state may be. The obtained microparticles can act as a microreactor if the outer shell is semi-porous to avoid any leakage of the core substance. Thus, the microencapsulation technology protects the active substance during the manufacturing of the smart material and brings up new functionality. Nevertheless, the functional performance of the microcapsules depends on the morphology, the chemical nature, and the surface characteristics of the polymeric shell influenced by the process parameters [9]. Thus, many factors such as monomers, prepolymers, and polymer nature and their concentration (1), emulsifiers concentration (2), stirring speed (3), temperature (4) were selected as parameters controlling the encapsulation efficiency [10]. The thermodynamic and kinetic parameters govern particle formation and its properties during the synthesis. In this context, the main microencapsulation processes used in the textiles fields can be divided into two main categories, i.e., (i) physicochemical processes, which include mainly simple and complex co-acervation and solvent evaporation, and (ii) chemical processes in which sol-gel processes, in situ and interfacial polymerization, are the leading used technics [3].

The choice of a particular process is determined by the solubility characteristics of the active compound, the shell material, and the desired shell properties [11]. Therefore, to design a semi-porous shell, interfacial 
polymerization appears as a suitable process. Among the various polycondensates, polyurethane-urea seems of particular interest with general characteristics by molecular composition [12]. In the water in oil polycondensation system to prepare polyurethane network, it is known that the reaction of isocyanate groups with water molecules during the capsule formation also forms urea crosslinking [13]. This formation depends on the water molecules diffusion through the polyurethane network and influences the thermal properties, controlling their permeation properties. The release rate of the active agent from the microparticles can also be affected by the shell's chemical nature, thickness, and mean diameter. Furthermore, Jabbari et al. have underlined that the porosity and the pore size of the surface were significantly affected by the amount of chain-extending agent [14]. The interfacial polycondensation reaction involves a step-growth polymerization between two monomers, each dissolved in a pair of immiscible phases. The reaction occurs at or in a thin region adjacent to the interface between the dispersed and continuous immiscible phases. The polymer product, being insoluble in both phases, accumulates as a film at the surface of contact between the two phases [15]. In our previous work, we have observed that the state of the surface of the microcapsules depends on the rate of polymer precipitation at the interface [6]. Thus, their surfaces are generally smooth when the polymer precipitates slowly due to the slow removal of the organic solvent. A resulting porous morphology was obtained with relatively faster precipitation of the polymer [11]. Furthermore, better thermal stability at high temperatures and thermal degradation were strongly influenced by the loading content and the surface roughness. On the other hand, the shell film microstructure and consequent properties such as film density and permeability are affected significantly by the polymer precipitation mechanism [16].

Nevertheless, the developed system implies using toluene as solvent of the continuous phase and taking into account the latest regulations in vigor and a concern of research of more "green" process. It is necessary to find an alternative to this solvent [17]. Therefore, in this work, Miglyol 812 (triglycerides of the fractionated plant fatty acids $\mathrm{C}_{8}$ and $\mathrm{C}_{10}$ ) was selected to be used in the continuous phase. The effects of the chosen solvent on the capsule morphology and the entrapment efficiency of water-soluble materials were investigated to determine the best chemical conditions to obtain microcapsules with good water permeation properties and better understand the influence of the continuous phase on the chemical and thermomechanical properties of the shell structure.

\section{EXPERIMENTAL}

\subsection{Materials}

Xylitol (Roquette Frères, content>99\%) $\left(\mathrm{C}_{5} \mathrm{H}_{12} \mathrm{O}_{5}\right)$, polyhydric alcohol was employed as the core material and used as a shell-forming monomer. Diphenyl methylene diisocyanate (MDI) (Suprasec 2030, Hüntsman ICl; blend of $\mathrm{MDI}$ isomers, 4,4'-diphenyl methylene diisocyanate principally) used as second shell-forming monomers was obtained from Aldrich and Huntsman ICl. Nonionic surfactant, Span $® 85$ (sorbitan trioleate), and poly (ethylene glycol)dioleate (PEG 400 dioleate) were purchased from Aldrich and used as an emulsifier. Toluene was of reagent grade and used without further purification. Miglyol $812 \mathrm{~N}$, a mixture of medium-chain triglycerides, was purchased from Sasol.

\subsection{Preparation of Microparticles}

According to the following method, the preparation of microparticles containing xylitol was carried out in a $250 \mathrm{ml}$ three-neck round-bottomed flask equipped with a mechanical stirrer via interfacial polymerization. $60 \mathrm{ml}$ of an aqueous solution containing $70 \%$-wt of xylitol previously solubilized in water at $80^{\circ} \mathrm{C}$ was emulsified in $120 \mathrm{ml}$ of an organic phase containing toluene or Miglyol and $4 \%$-wt of a binary mixture of PEG 400 dioleate and Span ${ }^{\circledR} 85$ at 13500 pm with a homogenizer (ultra turrax®, Ika, Germany). After 20 min, when the emulsion's expected droplet size was reached, the polymerization reaction was carried out by drop-wise addition of a solution containing from $20 \%$ wt of MDI in $10 \mathrm{ml}$ of toluene or Miglyol. The mixture was stirred continuously using a blade stirrer at 400 $\mathrm{rpm}$ at $80^{\circ} \mathrm{C}$ to complete the crosslinked polyurethane shell formation over 90 minutes. The resultant microparticles were recovered by filtration and washed once with the organic solvent to remove the remaining $\mathrm{MDI}$, and twice with water, and then dried at $60^{\circ} \mathrm{C}$ for one night.

The encapsulation yield was calculated as the recovered mass of microparticles and the weight of monomers and water introduced. It can be expressed as follows:

$$
\eta \quad(\%)=\frac{m_{\text {microparticles }}}{\sum m_{\text {monomers }}} \times 100
$$




\subsection{Characterizations}

\subsubsection{Chemical Analyses}

\subsubsection{Infrared Spectra Analyses}

The samples' chemical structure was analyzed by infrared spectra in an absorbance mode and recorded using Nicolet Nexus, connected to a PC. The number of scans was 256 , and the resolution was $0.5 \mathrm{~cm}^{-1}$. Samples were grounded and mixed with $\mathrm{KBr}$ to make pellets.

Their deconvolutions were performed using Peakfit 4.0 software (Jandel, San Rafael, CA) in the 1575-1800 $\mathrm{cm}^{-1}$ region into Gaussian peaks to analyze the FTIR spectra. These wavenumbers were used as initial parameters for curve fitting with Gaussian component peaks. Position, bandwidths, and amplitudes of the peaks were varied until (i) the resulting bands shifted by no more than $4 \mathrm{~cm}^{-1}$ from the initial parameters, (ii) all the peaks had reasonable half-widths $\left(<20-25 \mathrm{~cm}^{-}\right.$ ${ }^{1}$ ), and (iii) good agreement between the calculated sum of all components and the experimental spectra was achieved $\left(r^{2}>0.99\right)$. The results of four independent experiments were averaged.

\subsubsection{Solid-State NMR}

Solid-state NMR: 13C NMR measurements were performed on a Bruker ASX 100 low field spectrometer at $25.2 \mathrm{MHz}$ with magic angle spinning (MAS), with high power $1 \mathrm{H}$ decoupling and $1 \mathrm{H}-13 \mathrm{C}$ crosspolarization (CP) using a $7 \mathrm{~mm}$ Bruker probe. The Hartmann-Hahn matching condition was obtained by adjusting the power on the $1 \mathrm{H}$ channel for a maximum 13C FID signal of adamantane. All spectra were acquired with a contact time of $1 \mathrm{~ms}$. The repetition time was $5 \mathrm{~s}$, and the reference used was tetramethylsilane (TMS).

\subsubsection{Thermal Analyses}

\subsubsection{Differential Scanning Calorimetry}

The particles' thermal behavior was recorded using a TA instrument type DSC 2920 piloted on PC with TA Advantage control software. Indium was used as a standard for temperature calibration, and the analysis was made under a constant stream of nitrogen (50 ml.min-1). Samples were placed in aluminum pans which were hermetically sealed before being placed on the calorimeter thermocouples. The sample space was purged with nitrogen at a constant flow (50 ml.min-1) during the experiments, and the temperature range was from -30 to $150^{\circ} \mathrm{C}$. Transition temperatures and enthalpies were obtained by averaging the results of a series of four independent experiments on $(5.0 \pm 0.2$ $\mathrm{mg}$ ) samples with a scanning speed of $1 \mathrm{~K} \cdot \mathrm{min}^{-1}$.

The content of xylitol in the microparticles can be estimated according to the measured enthalpy:

$$
\text { xylitol content }=\frac{\Delta H_{\text {microparticles }}}{\Delta H_{\text {xylitol }}^{0}} \times 100 \%
$$

Where $\Delta H_{\text {microparticles }}$ and $\Delta H_{x y l i t o l}^{0}$ are the melting enthalpy of microparticles and xylitol, respectively.

\subsubsection{Enthalpy of Dilution}

The absorption of water, its mass transfer in the microparticles, and the enthalpies of dilution were determined with an isothermal calorimeter Setaram C80, equipped with a reversible mixing cell. A determined weight (ms) of raw xylitol (or microparticles) was introduced in the bottom part of the mixing cell closed by a stopper, a determined amount of distilled water (mw; $\mathrm{mw}=\mathrm{ms}$ for $x y l i t o l$ sample; and $\mathrm{mw}=\mathrm{ms} / 2$ for microparticles samples) was then added. A mass $(\mathrm{ms}+\mathrm{MW})$ of water was introduced in the reference cell. The two compartments were introduced into the calorimeter, and when the baseline was stable, the acquisition was started for $3 \mathrm{hr}$. After approximately 12 minutes, C80 was mechanically turned over ten times to mix water and the powder at $35^{\circ} \mathrm{C}$. The active loading content of xylitol in the microparticles can be estimated according to the measured enthalpy of dilution:

active xylitol $\quad(\%)=\frac{\Delta_{\text {dil }} H_{\text {microparticles }}}{\Delta_{\text {dil }} H_{\text {xylitol }}^{0}} \times 100 \%$

Where $\Delta_{\text {dil }} H_{\text {microparticles }}$ and $\Delta_{\text {dil }} H_{x y l i t o l}^{0}$ are the melting enthalpy of microparticles and xylitol (161.3 J.g ${ }^{1}$ ), respectively.

\subsubsection{Mean Diameter and Morphology of the Microparticles}

At least 200 microparticles were picked up from the optical microscope photographs (OM, Axiolab Polar Carl Zeiss) using a $40 \times$ objective lens. The diameters were measured to obtain the average number diameter of the microparticles. The photographs were captured using the Perfect Image-Vision Clara. Three points in the circular shape of each particle observed were pointed to obtain the mean diameter. The form of the 
microparticles was also observed by scanning electron microscopy (SEM) (Philips XL30 ESEM / EDAXSAPPHIRE).

\section{RESULTS AND DISCUSSION}

\subsection{Emulsion Step}

\subsubsection{Choice of Solvent for the Continuous Phase}

The solubility and the amount of xylitol introduced in the dispersed phase can be optimized by the temperature rise of the binary mixture water/xylitol [18]. The synthesis temperature is chosen according to the solubility of xylitol near its melting point. It was decided to regulate all the syntheses in a glycol bath at $80^{\circ} \mathrm{C}$. The choice of the solvents being appropriate for the formulation of the continuous phase was established starting from the physicochemical properties of those while considering the solubility of the MDI. The selection criteria were not only based on the analysis of the solubility parameters of the different compounds (solvents, polyurea urethane membrane), but also by considering the stability of the emulsion, and more particularly, the existence of the phenomena of creaming or sedimentation, induced by the difference in density between the known medium and the dispersed medium. The importance of this phenomenon can be illustrated by Stoke's law showing the rate of destabilization of an emulsion by equating gravitational force with the opposing hydrodynamic force [19]. Thus, the lower the sedimentation rate, the more stable the system. Based on obtaining particles with an average diameter of $10 \mu \mathrm{m}$, for a set of organic solvents. The calculation allowed us to define that the lowest sedimentation speeds are obtained in the case of Miglyol $\left(0.03 \times 10^{-4} \mathrm{~m} . \mathrm{s}^{-1}\right)$, ethanol $\left(1.16 \times 10^{-4} \mathrm{~m} . \mathrm{s}^{-1}\right)$, and toluene $\left(1.97 \times 10^{-4} \mathrm{~m} . \mathrm{s}^{-1}\right)$. Miglyol and toluene were chosen because of their higher boiling point. The emulsion type is also determined by the volume ratio between the dispersed and continuous phases [20]. To meet this criterion for this, and according to Maa et al. [21], it is fixed at $1 / 2$.

\subsubsection{Dispersed Phase Formulation}

The establishment of a stable emulsion plays an essential role in obtaining microparticles. It depends on many factors whose importance varies from one system to another in the function of the desired characteristics. In this study, the temperature of the emulsion step is related to the solubilization temperature of the active ingredient. Miscibility tests have shown that water is a non-solvent for polyisocyanates and a solvent for polyols, such as xylitol [22]. The use of water allows decreasing the temperature, viscosity, and density of the dispersed phase while avoiding the destabilization phenomena linked to external reagents acting as nucleating agents of xylitol. Water also allows the creation of a poly (ureaurethane) network [23].

Particle formation during the homogenization depends on a stress balance between the turbulent forces tending to break up the droplet and the forces from interfacial tension holding a droplet together. The degree of a liquid droplet breakup is led by three forces; surface tension, which tries to maintain the spherical shape, while viscous and inertial forces attempt to deform the droplet. The droplet size is determined by a balance between the droplet breakup and the coalescence forces. These two factors are considered the most critical phenomena, which influence the droplet size and size distribution during the emulsification process [24]. Figure depicts the variations of the phase viscosity ratio as a function of the mole fraction of xylitol in the dispersed phase.

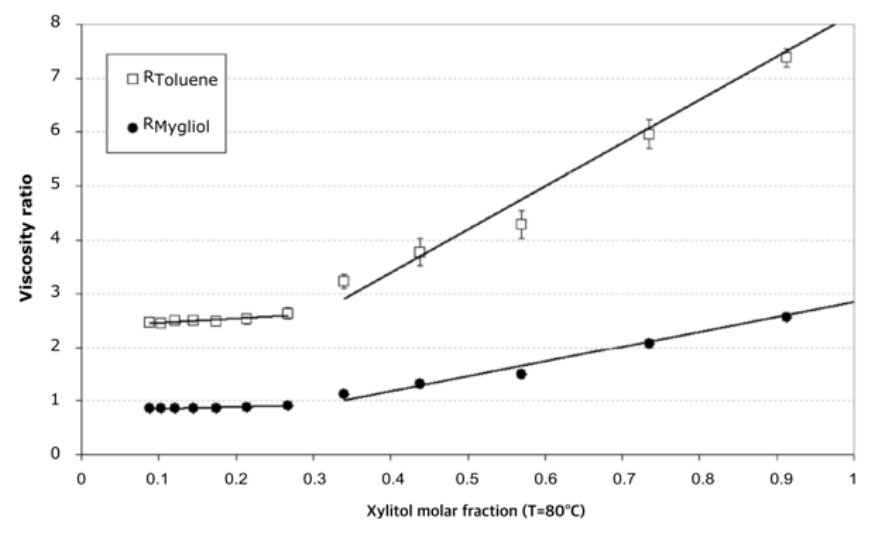

Figure 1: Effect of xylitol amount on the viscosity ratio of the dispersed to the continuous phases.

The estimation of $R$ provides insights into the fractionation mechanism of the aqueous dispersed phase. The viscosity ratio $R_{\text {Toluene }}$ is stable in the toluene, reaching an average value of 2.5 in a range of mole fractions between 0.1 and 0.26 . It indicates that the dispersed phase aqueous dispersed phase is more viscous than the dispersing phase composed of toluene $\left(R_{\text {Toluene }}>1\right)$. From a molar fraction of 0.3 , $\mathrm{R}_{\text {Toluene }}$ increases strongly to reach a value higher than 7 for a Xylitol molar fraction of 0.9 . $R_{\text {Mygliol }}$ value is constant up to a molar fraction of about 0.26 and increases more slowly than $\mathrm{R}_{\text {Toluene }}$ from 0.3 to 2.8 . As seen in Figure, an identical shape of curves was obtained whatever the organic solvent used. A slope 
failure occurs from xylitol to water molar ratio of 0.3 . From this point, an increase of xylitol induces a slight increase in viscosity ratio, which limits the shearing of the two phases. Furthermore, we can notice that the viscosity ratio using toluene is higher than when Miglyol was used. Moreover, it is not surprising to observe a lower mean diameter value for Miglyol than for Toluene (Figure 2). Therefore, the stability of the xylitol droplets should be enhanced with a low R-value. Thus, an Rvalue of 0.26 was chosen to design the microencapsulation process.

\subsubsection{Effect of Surfactant Formulation}

The approach used to formulate the stable w/o emulsion was based on the concept of required hydrophilic-lipophilic balance (HLB), in accordance with the Griffin's (1949) concept [25, 26].

Mixtures of Span® 85 and PEG 400 dioleate in different proportions were used to cover the range in HLB values from 1.8 to 8.3. In these experiments, a $70 \%$ aqueous Xylitol solution was emulsified at $80^{\circ} \mathrm{C}$ under a high shear rate $(13500 \mathrm{rpm})$ in a lipophilic phase containing toluene or Miglyol and the surfactant mixture. Emulsion stability was judged by the appearance and by observing phase separation, if any, by bare eye observation and under microscope 1.5 hours after preparation. The type of emulsion and the mean diameter and particle size distribution differed according to the HLB value of the surfactant mixture tested (Table 1 and Figure ). In most cases, well- defined water in oil emulsion was obtained with a low mean diameter and good stability for an HLB value of 3 for toluene and 7 for Mygliol. For high HLB values, between 6 and 8.3 in the case of toluene and 8.3 with Miglyol, multiple unstable emulsions were obtained, not allowing the formation of microparticles in satisfactory conditions. Thus, an HLB value of 3 was chosen to realize the microencapsulation synthesis with toluene and 7 with Miglyol. Depending on the solvent used, the HLB required to achieve good stability and narrow size distribution during emulsification is not the same. The higher density of Miglyol tends to decrease the sedimentation phenomenon. The droplet diameter evolution is consistent with the measured viscosities since the smaller ones were observed in the emulsion with Miglyol.

\subsection{Formation of Poly(Urea-Urethane) Microparticles}

\subsubsection{Mechanism of Shell Formation}

When microparticles are prepared by interfacial polymerization, the shell's physicochemical properties depend not only on its chemical nature but also on all experimental parameters of the process. Indeed, the solvent used in the dispersed phase is a suitable solvent for the starting monomer (xylitol) but acts as a non-solvent for the produced polymer. Thus, the formation of the membrane around the droplets is governed by the swelling power of both phases, and a porous structure may be obtained when the solvency of the medium for the polymer is low. Furthermore, the

Table 1: Influence of the HLB Values of Span 85/Dioleate PEG 400 Mixture on the Emulsion Type, Stability, and Particle Size Distribution

\begin{tabular}{|c|c|c|c|c|}
\hline Organic Solvent & $\begin{array}{c}\text { HLB of Surfactants } \\
\begin{array}{c}\text { Span }{ }^{\circledR} 85 \text { / Dioleate PEG } 400 \\
\text { (wt-\%/wt-\%) }\end{array}\end{array}$ & \multicolumn{3}{|c|}{ Emulsion } \\
\hline Toluene & $3(81.5 / 18.5)$ & w/o & +++ & $13.0 \pm 1.5$ \\
\hline Toluene & $4(66 / 34)$ & w/o & +++ & $15.6 \pm 2.3$ \\
\hline Toluene & $6(35.4 / 64.6)$ & w/o & - (coalescence) & $15.3 \pm 4.5$ \\
\hline Toluene & $7(20 / 80)$ & o/w/o & -- (sedimentation) & $20.6 \pm 8.6$ \\
\hline Toluene & $8.3(0 / 100)$ & o/w/o & -- (sedimentation) & $25.7 \pm 10.8$ \\
\hline Miglyol & $5(50.8 / 49.2)$ & o/w/o & $+($ coalescence $)$ & $12.9 \pm 4.2$ \\
\hline
\end{tabular}

${ }^{\text {a }}$ Classification: +++ , excellent ; ++, good ; +, satisfactory ; -, poor ; --, very poor.

${ }^{\mathrm{b}}$ Particle diameters were expressed as an average value of 200 measurements determined by optical microscopy. 


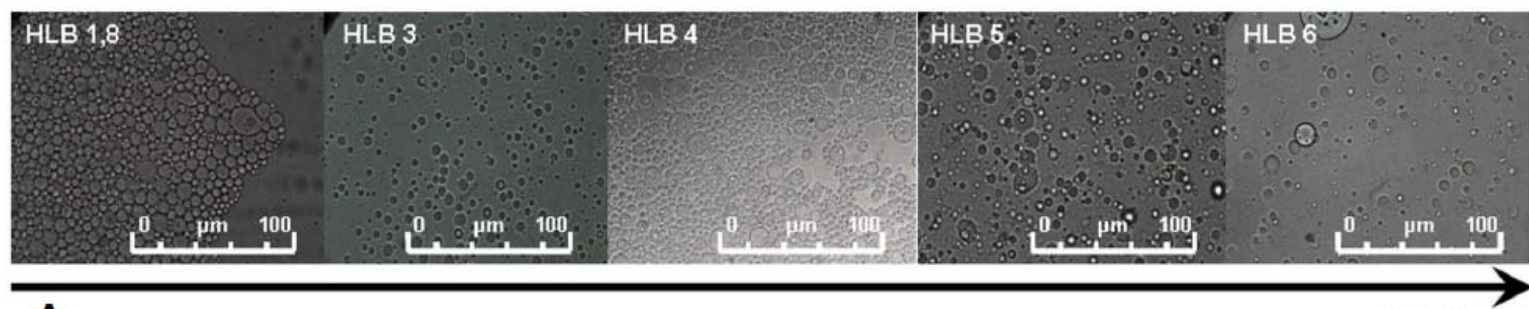

A

HLB

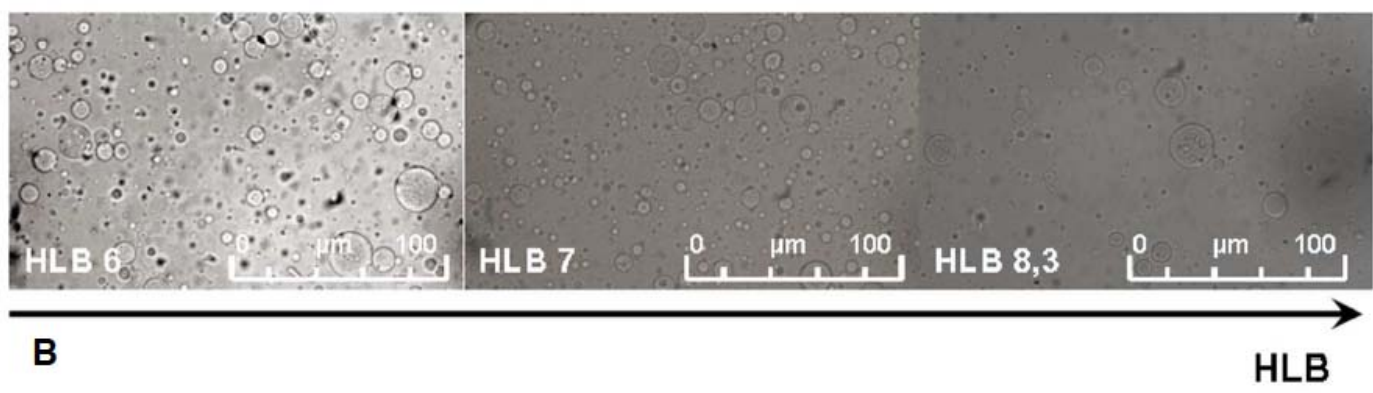

Figure 2: Effect of HLB value on emulsion properties according to the solvent used ( $\mathrm{A}$ - toluene, $\mathrm{B}$ - Miglyol).

particle morphology is influenced by the rate of precipitation of the polymer, and a higher rate promotes the formation of the less uniform and more porous membrane. Therefore, the knowledge of the mechanism of the shell formation and polycondensation reaction that occurs during the synthesis is necessary to control the shape of the particles and morphology. The polycondensation mechanism between the isocyanate and hydroxyl functions at the droplet interface is illustrated through the following reaction schemes (Figure ). In the first reaction, MDI monomers can be slowly hydrolyzed at the interface to form an unstable amino acid group that dissociates in the amine end group and carbon dioxide [27]. This reaction, occurring at the oil side of the interface, depends mainly on the water molecules diffusion in the medium or through the polymer shell network. The formation and release of $\mathrm{CO}_{2}$ during the membrane formation contribute to increasing the porosity of the particles. The amine end group reacts with an isocyanate end group to form a urea linkage in the second reaction. In the third reaction, diisocyanate or isocyanate end groups react with hydroxyl groups of xylitol to form a urethane linkage and, therefore, the polyurethane chains. Other reactions can occur during the shell growth. The reaction between an isocyanate end and urethane or urea unit results in an allophanate or a biuret, respectively, creating interconnections between the polymer chains. Furthermore, the crosslinking reactions and network formation were also enhanced by the monomer functionality, 2.3 for MDI and 5 for xylitol, respectively.

\subsubsection{Effect of the Solvent Used and Monomers Amount of Urea and Urethane Linkages Formation}

The choice of solvent and the xylitol/MDI ratio play a significant role in forming the membrane. From Table 2, it can be observed that the increase of MDI leads to a rise in urea groups, whatever the solvent used. Thus, the isocyanate functions react more quickly with water molecules than the hydroxyl groups of xylitol due to their presence at the interface, and therefore the probability of reaction increases [28].

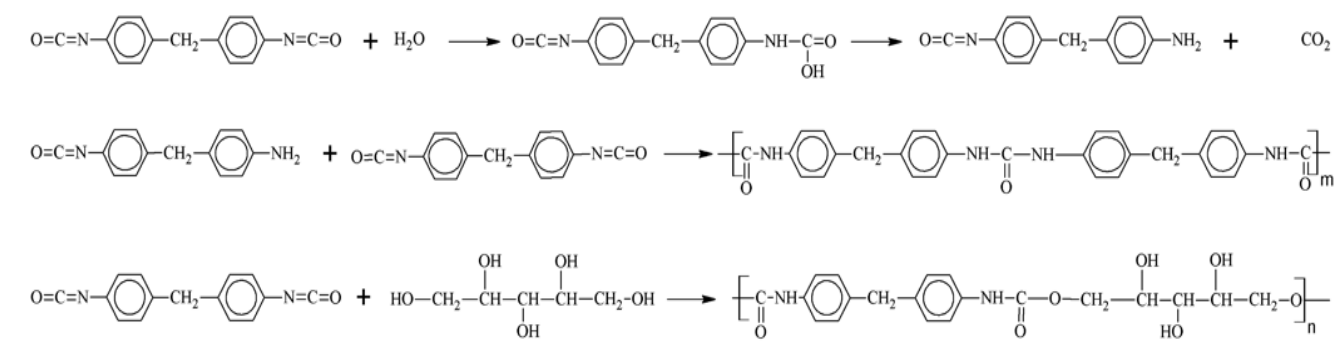

Figure 3: Reaction schemes of poly(urea-urethane) shell. 
When the proportions in MDI vary between 16.6 and $23.0 \%$, the use of Miglyol promotes the formation of urethane groups, whereas the content of urea groups is higher with toluene (Table 2). This phenomenon is related to the diffusion of the aqueous monomer and the water molecules at the interface. The presence of Miglyol acts as a barrier at the interface, whereas the water molecules can diffuse slightly, inducing the formation of urea groups in toluene. Nevertheless, a higher content in MDI promotes the formation of urea linkages in Miglyol due to the reactions with water or surfactant molecules, which lead to the formation of soluble oligomers. Whereas in toluene, MDI can react with the amino groups.

Thus, the quantitative analysis of the urea-urethane region of FTIR and NMR spectra by peak deconvolution allows to determine that the effect of the used solvent is also related to the solubility of the primary oligomers in the continuous medium, and therefore to the composition of the obtained shell. During the shell formation step, the polycondensation reactions lead to an increase of the viscosity at the interface and the continuous medium due to the soluble diffusion of the soluble oligomers. The phenomena limit the diffusion of the xylitol molecules and therefore induces a decrease in the urethane bond formation.

Table 2: FT-IR Analyses, Allowing a Qualitative Description of the Shell Structure with the Chemical Function Present in the Polymer, and 13C NMR Spectroscopy Measurements were Performed to Provide Supplementary Information about the Chemical Structure of the Polymeric Shell Formed Around Xylitol Droplet

\begin{tabular}{|c|c|c|c|c|}
\hline \multirow{2}{*}{$\begin{array}{c}\text { Sample } \\
\text { Code }\end{array}$} & \multicolumn{2}{|c|}{ FTIR } & \multicolumn{2}{c|}{ NMR } \\
\cline { 2 - 5 } & $\begin{array}{c}\text { Urethane } \\
\text { (\%) }\end{array}$ & $\begin{array}{c}\text { Urea } \\
\text { (\%) }\end{array}$ & $\begin{array}{c}\text { Urethane } \\
\text { (\%) }\end{array}$ & $\begin{array}{c}\text { Urea } \\
\text { (\%) }\end{array}$ \\
\hline \hline ST1 & 44.8 & 55.2 & 42.4 & 57.6 \\
\hline SM1 & 70.1 & 29.9 & 72.2 & 27.8 \\
\hline ST2 & 34.7 & 65.3 & 33.0 & 67.0 \\
\hline SM2 & 59.1 & 40.9 & 57.0 & 43.0 \\
\hline ST3 & 37.0 & 63.0 & 33.0 & 67.0 \\
\hline SM3 & 29.4 & 70.6 & 25.0 & 75.0 \\
\hline
\end{tabular}

\subsubsection{Effect of the Solvent on the Particles Size Distribution and Encapsulation Yield}

The continuous phase solvent modifies the kinetics of the polymerization reactions and the formation of the microcapsules shell. Furthermore, the increased viscosity in the toluene phase results in the decrease of the mean diameter due to the coupled effect of stirring and the solubility of the oligomers. Indeed, a part of them, which does not participate in the formation of the membrane, remains in the continuous medium. As a result, the encapsulation yield with the use of toluene is lower than with Miglyol. The increase of MDI content allows increasing it, but it is lower than the Miglyol one. All these observations lead to the fact that the polymerization reactions are located at the interface of the Miglyol system, like a homogeneous system. On the other hand, the use of toluene leads to a heterogeneous polymerization, contributing to the diffusion of oligomers in the solvent that does not form the membrane. Furthermore, taking into consideration the solubility parameters of the polymer and solvents, a much lower solubility of the polymer membrane in toluene than in Miglyol leads to early precipitation of the polymer and lower permeability. Thus, the diffusion of xylitol to the organic phase is impeded. [29].

The particle size distribution and the mean diameter of the obtained microparticles depend on the xylitol/MDI ratio and the solvent used. The use of Miglyol leads to a lower mean diameter than toluene, which is due to the emulsion properties and the shell mechanism formation. According to the MDI content, the mean diameter seems to be constant (considering the standard deviation). The slight decrease correlated to the increase of the encapsulation yield is related to the increase in the surface area contact, which allows for better consumption of the monomers.\#

Therefore, the mean diameter was found between 9.8 and $19.6 \mu \mathrm{m}$ and depended on the MDI introduced and the organic solvent used during the encapsulation stages (Table 3). Thus, it was not surprising to observe that the use of Miglyol rather than toluene allows decreasing the mean diameter. On the other hand, adding MDI contributed to an increase of the viscosity of the continuous phase, which improved the shearing of the dispersed phase and led to the formation of smaller microparticles. The reduction in droplet size and the increase in their surface area with a higher stirring rate contribute to the rapid formation of the urea linkage in the shell. Then, the formation of the porous membrane is controlled by the diffusion of the monomers at the interface and therefore linked to the primary membrane permeability. The addition of MDI allowed the formation of the crosslinked network and delayed xylitol diffusion through the polymer membrane. Thus, the formation of a dense primary membrane decreased the growth of the shell. 
Furthermore, the encapsulation yield increases with an increase of MDI in the Miglyol system rather than in the toluene one. According to the solvent used, the microparticles morphology varies a lot. They exhibited poor sphericity and were mostly irregular for a low amount of MDI. The morphology is related to the amount of isocyanate function yielded insufficiently rigid particles, and leading to irregular collapse. The use of toluene leads to the formation of a spongy surface and porous wall. In contrast, with Miglyol, a raspberry-like morphology is obtained with the presence of tiny particles onto the surface (Figure ). The reason is due to excessive oligomer attached to the surface of the micelles, leading to the formation of an irregular surface state [30].

The introduction of MDI into the continuous phase induces the formation of the oligomers at the interface. On the one hand, if the oligomers are soluble in the dispersed phase, they diffused in the droplet, and further reactions lead to the formation of a matrix structure. On the other hand, when oligomers are insoluble in the dispersed phase, they concentrate at the raspberry-liked core-shell structure. Thus, it was obtained with a higher amount of MDI.

\subsection{Loading Content and Permeation Properties}

The thermal characterizations were used to determine the loading content and the active loading content, meaning the xylitol amount reacting with water molecules. From the results listed in Table 4, microcapsules have a semi-porous shell and a sufficient free volume to undergo the endothermic dissolution reaction with water. The measured values of xylitol melting enthalpy and heat of dissolution were found at about $248.7 \mathrm{~J} . \mathrm{g}^{-1}$ and $167.1{\mathrm{~J} . \mathrm{g}^{-1}}^{-1}$ respectively.

In the toluene system, the xylitol loading content is related to the amount of MDI adding, urethane bonds, and the encapsulation yield. The increase in urethane group content corresponds to a more significant number of reactions between the isocyanate and hydroxyl functions, resulting in fewer xylitol molecules that can subsequently crystallize. Thus, the higher crystalline xylitol content was found for ST2, having the lower urethane content. The proportion of active xylitol is related to the amount of xylitol accessible to promote water reaction and the obtained morphology. For the samples ST1 and ST3, it is proportional to the crystalline xylitol content, indicating that a part of xylitol

Table 3: Formulation of Poly(Urea-Urethane) Microcapsules. Influence of the Amounts of Monomers and Stirring Rate on Encapsulation Yield and Microcapsules Mean Diameter

\begin{tabular}{|c|c|c|c|c|c|}
\hline \multirow[b]{2}{*}{ Sample Code } & \multicolumn{2}{|c|}{ Amount of Monomers } & \multirow[b]{2}{*}{ Organic Solvent } & \multirow{2}{*}{$\begin{array}{c}\text { Encapsulation } \\
\text { Yield } \\
(\%)\end{array}$} & \multirow{2}{*}{$\begin{array}{c}\text { Microcapsules } \\
\text { Mean Diameter } \\
(\mu \mathrm{m})\end{array}$} \\
\hline & $\begin{array}{l}\text { Xylitol } \\
\text { (\%-wt) }\end{array}$ & $\begin{array}{c}\text { MDI } \\
\text { (\%-wt) }\end{array}$ & & & \\
\hline SM1 & 83.4 & 16.6 & Miglyol & 98.5 & $9.8 \pm 1.2$ \\
\hline ST2 & 77.0 & 23.0 & Toluene & 74.7 & $15.3 \pm 2.0$ \\
\hline ST3 & 71.5 & 28.5 & Toluene & 51.8 & $16.6 \pm 2.4$ \\
\hline SM3 & 71.5 & 28.5 & Miglyol & 99.0 & $12.3 \pm 1.9$ \\
\hline
\end{tabular}
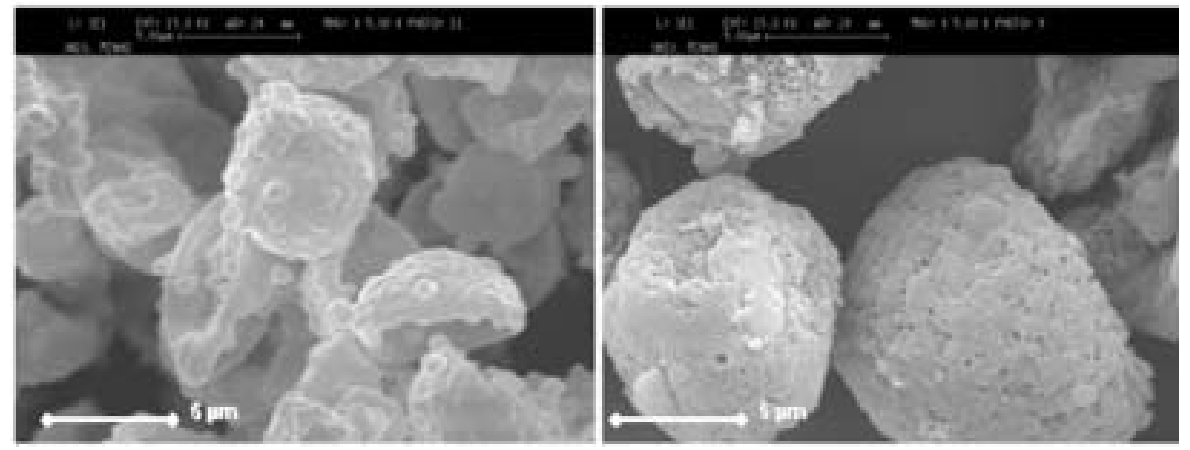

Figure 4: SEM micrographs of microparticles obtained from synthesis labeled SM2 (left) and ST2 (right). 
Table 4: Thermal Properties of the Obtained Microcapsules

\begin{tabular}{|c|c|c|c|c|c|}
\hline Sample Code & \multicolumn{2}{|r|}{ DSC } & $\begin{array}{l}\text { Heat of Dilution } \\
\text { at } 35^{\circ} \mathrm{C}\left(\mathrm{J} \cdot \mathrm{g}^{-1}\right)\end{array}$ & $\begin{array}{l}\text { Active Xylitol } \\
\text { Content (\%) }\end{array}$ & $\begin{array}{c}\text { Ratio Activel } \\
\text { Crystalline Xylitol }\end{array}$ \\
\hline SM1 & 178.6 & 69.0 & 18.8 & 11.7 & 0.17 \\
\hline ST2 & 196.3 & 75.8 & 124.3 & 77.1 & 1.01 \\
\hline ST3 & 140.7 & 54.3 & 38.1 & 23.6 & 0.43 \\
\hline SM3 & 108.4 & 41.9 & 45.1 & 27.9 & 0.66 \\
\hline
\end{tabular}

is not involved in the reaction, maybe due to a matrix structure, which hindered the diffusion of the water molecules in this polymer network. The calculated ratio active to crystalline xylitol for the sample ST2 is close to 1 , in this case, a core/shell was obtained, and all the free xylitol can undergo the reaction with water.

The use of the Miglyol as the solvent for the continuous phase modified the properties of the microcapsules. The crystalline xylitol content in the capsules is not proportional to the urethane content, indicating that more than one hydroxyl group from the xylitol molecules has reacted with the isocyanate to crosslink the shell. The active xylitol content increases with the decrease of urethane content and the ratio of active to crystalline xylitol. Lower ratios are due to the formation of microspheres and the presence of tiny particles on their surface. The roughness of the membrane and the lower porosity is not suitable for the water molecules transfer. The obtained morphology is close to a microcapsule for the higher ratio, promoting an endothermic reaction. Besides, the presence of Mygliol molecules adsorbed on the surface of the microcapsules was experimentally detected, which may be a barrier to the transfer of water molecules, limiting the detection of active xylitol.

\section{CONCLUSION}

The study of the preparation of poly(urea-urethane) microparticles from isocyanate and xylitol shows that the synthesis parameters, especially the MDI amount and the organic solvent choice, lead to the formation of two distinguished morphologies. This difference can be related to the formed oligomers' solubility parameters during the first stage of the polycondensation and the solvent-oligomer interaction. Furthermore, the continuous phase's solvent also influences the encapsulation yield, the mean diameter of the particles, and the shell formation mechanism. Furthermore, excepted for one sample, it was noticed that all the entrapped xylitol could not undergo reaction with water, leading to poor permeation properties. Nevertheless, the use of Miglyol seems to be an excellent way to replace toluene in this process without considering the thermal properties of these microparticles. To optimize the Mygliol system, additional studies need to be conducted, including post-treatment, to find a suitable way to remove the solvent from the pores of the capsule shell.

\section{REFERENCES}

[1] Reyes-Ortega F, Hosseini M. Smart Polymeric-Based Microencapsulation: A Promising Synergic Combination. In Hosseini M, Makhlouf ASH, editors. Industrial Applications for Intelligent Polymers and Coatings2016. p. 577-604. https://doi.org/10.1007/978-3-319-26893-4 27

[2] Boh B, Sumiga B. Microencapsulation technology and its applications in building construction materials. Materials and Geoenvironment. 2008;55(3):329-44.

[3] Salaün F. Microencapsulation technology for smart textile coatings. In: Hu J, editor. Active Coatings for Smart Textiles: Woodhead Publishing; 2016. p. 179-220. https://doi.org/10.1016/B978-0-08-100263-6.00009-5

[4] Farooq AS, Zhang P. Fundamentals, materials and strategies for personal thermal management by next-generation textiles. Composites Part A: Applied Science and Manufacturing. https://doi.org/10.1016/j.compositesa.2020.106249

[5] Bedek G, Salaün F, Martinkovska Z, Devaux E, Dupont D. Evaluation of thermal and moisture management properties on knitted fabrics and comparison with a physiological model in warm conditions. Appl Ergon. 2011;42(6):792-800. https://doi.org/10.1016/j.apergo.2011.01.001

[6] Salaün F, Bedek G, Devaux E, Dupont D, Gengembre L. Microencapsulation of a cooling agent by interfacial polymerization: Influence of the parameters of encapsulation on poly(urethane-urea) microparticles characteristics. J $\begin{array}{ll}\text { Membrane } & \text { Sci. }\end{array}$ https://doi.org/10.1016/j.memsci.2010.11.033

[7] Salaün F, Bedek G, Devaux E, Dupont D, Deranton D. Investigation of water absorption and diffusion in microparticles containing xylitol to provide a cooling effect by thermal analysis. Int $\mathrm{J}$ Thermophys. 2009;30(4):1242-56. https://doi.org/10.1007/s10765-009-0649-4 
[8] Delgado Arcaño Y, Valmaña García OD, Mandelli D, Carvalho WA, Magalhães Pontes LA. Xylitol: A review on the progress and challenges of its production by chemical route. Catal Today. 2020;344:2-14. https://doi.org/10.1016/j.cattod.2018.07.060

[9] Petrulis D, Petrulyte $S$. Potential use of microcapsules in manufacture of fibrous products: A review. J Appl Polym Sci. 2018;136(7).

https://doi.org/10.1002/app.47066

[10] Salaün F. Microencapsulation by Interfacial Polymerization. In: Mittal V, editor. Encapsulation Nanotechnologies. Hoboken, NJ, USA: John Wiley \& Sons, Inc.; 2013. p. 13773.

https://doi.org/10.1002/9781118729175.ch5

[11] Perignon C, Ongmayeb G, Neufeld R, Frere Y, Poncelet D. Microencapsulation by interfacial polymerisation: membrane formation and structure. J Microencapsul. 2015;32(1):1-15. https://doi.org/10.3109/02652048.2014.950711

[12] Ben Abdelkader M, Azizi N, Baffoun A, Chevalier Y, Majdoub M. New microcapsules based on isosorbide for cosmetotextile: Preparation and characterization. Ind Crop Prod. 2018;123:591-9. https://doi.org/10.1016/j.indcrop.2018.07.020

[13] Silva M, Martins I, Barreiro F, Dias M, Rodrigues AE. Preparation and characterization of poly(urethane-urea) microcapsules containing limonene. Kinetic analysis. Int $\mathrm{J}$ Polym Anal Ch. 2017;22(8):709-24. https://doi.org/10.1080/1023666X.2017.1369253

[14] Jabbari E, Khakpour M. Morphology of and release behavior from porous polyurethane microspheres. Biomaterials. 2000;21(20):2073-9. https://doi.org/10.1016/S0142-9612(00)00135-6

[15] Frere Y, Danicher L. Microencapsulation par polycondensation inter-faciale. In: Vandamme T, Poncelet $D$, Subra-Paternault $P$, editors. Microencapsulation: des sciences aux technologies. Paris, France: Librairie Lavoisier; 2007. p. 54-69.

[16] Ricardo F, Pradilla D, Luiz R, Alvarez Solano OA. A MultiScale Approach to Microencapsulation by Interfacial

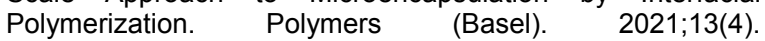
https://doi.org/10.3390/polym13040644

[17] Alizadegan F, Pazokifard S, Mirabedini SM, Danaei M, Farnood R. Polyurethane-based microcapsules containing reactive isocyanate compounds: Study on preparation procedure and solvent replacement. Colloids and Surfaces A: Physicochemical and Engineering Aspects. 2017;529:750-9. https://doi.org/10.1016/j.colsurfa.2017.06.058

[18] Held C, Sadowski G, Carneiro A, Rodríguez O, Macedo EA. Modeling thermodynamic properties of aqueous single-solute and multi-solute sugar solutions with PC-SAFT. Aiche J. 2013;59(12):4794-805.

https://doi.org/10.1002/aic.14212

[19] Goodarzi F, Zendehboudi S. A Comprehensive Review on Emulsions and Emulsion Stability in Chemical and Energy
Industries. The Canadian Journal of Chemical Engineering. 2018;97(1):281-309.

\section{https://doi.org/10.1002/cjce.23336}

[20] Piacentini E. Emulsion. Encyclopedia of Membranes2016. p. 679-82.

https://doi.org/10.1007/978-3-662-44324-8 1066

[21] Maa YF, Hsu C. Liquid-liquid emulsification by rotor/stator homogenization. J Control Release. 1996;38:219-28. https://doi.org/10.1016/0168-3659(95)00123-9

[22] Wang S, Li Q-S, Li Z, Su M-G. Solubility of Xylitol in Ethanol, Acetone,N,N-Dimethylformamide, 1-Butanol, 1-Pentanol, Toluene, 2-Propanol, and Water. Journal of Chemical \& Engineering Data. https://doi.org/10.1021/je060348v

[23] Saihi D, Vroman I, Giraud S, Bourbigot S. Microencapsulation of ammonium phosphate with a polyurethane shell. Part II. Interfacial polymerization technique. Reactive and Functional Polymers. 2006;66(10):1118-25.

https://doi.org/10.1016/j.reactfunctpolym.2006.02.001

[24] Leiva J, Geffroy E. Evolution of the Size Distribution of an Emulsion under a Simple Shear Flow. Fluids. 2018;3(3). https://doi.org/10.3390/fluids3030046

[25] Pensé AM, Vauthier C, Puisieux F, Benoit JP. Microencapsulation of benzalkonium chloride. International Journal of Pharmaceutics. 1992;81(2-3):111-7. https://doi.org/10.1016/0378-5173(92)90002-J

[26] Schmidts T, Schlupp P, Gross A, Dobler D, Runkel F. Required HLB Determination of Some Pharmaceutical Oils in Submicron Emulsions. J Disper Sci Technol. 2012;33(6):81620.

https://doi.org/10.1080/01932691.2011.584800

[27] Attaei M, Loureiro MV, Do Vale M, Condeco JAD, Pinho I, Bordado JC, et al. Isophorone Diisocyanate (IPDI) Microencapsulation for Mono-Component Adhesives: Effect of the Active $\mathrm{H}$ and NCO Sources. Polymers (Basel). 2018;10(8).

https://doi.org/10.3390/polym10080825

[28] Raaijmakers MJT, Benes NE. Current trends in interfacial polymerization chemistry. Prog Polym Sci. 2016;63:86-142. https://doi.org/10.1016/i.progpolymsci.2016.06.004

[29] Gaudin F, Sintes-Zydowicz N. Correlation between the polymerization kinetics and the chemical structure of poly(urethane-urea) nanocapsule membrane obtained by interfacial step polymerization in miniemulsion. Colloids and Surfaces A: Physicochemical and Engineering Aspects. 2012;415:328-42.

https://doi.org/10.1016/j.colsurfa.2012.09.040

[30] Shao J, Yu C, Bian F, Zeng Y, Zhang F. Preparation and Properties of Hydrophilic Rosin-Based Aromatic Polyurethane Microspheres. ACS Omega. 2019;4(2):2493-9. https://doi.org/10.1021/acsomega.8b03334

\section{DOI: https://doi.org/10.15377/2409-983X.2020.07.4}

(c) 2020 F. Salaün; Avanti Publishers.

This is an open access article licensed under the terms of the Creative Commons Attribution Non-Commercial License (http://creativecommons.org/licenses/by-nc/3.0/) which permits unrestricted, non-commercial use, distribution and reproduction in any medium, provided the work is properly cited. 